\title{
Comparing Diagnostic Evaluations for Rectal Bleeding and Breast Lumps in Primary Care: a Retrospective Cohort Study
}

\author{
Lydia E. Pace, MD, MPH ${ }^{1,2}$, Sanja Percac-Lima, MD, PhD ${ }^{1,3}$, Kevin H. Nguyen, $\mathrm{MS}^{4}$, \\ Charis N. Crofton, BA ${ }^{5}$, Katharine A. Normandin, BS ${ }^{5}$, Sara J. Singer, MBA, $P h D^{4,6}$, \\ Meredith B. Rosenthal, $P h D^{4}$, and Alyna T. Chien, $M D, M S^{1,5}$
}

\begin{abstract}
'Harvard Medical School, Boston, MA, USA; ${ }^{2}$ Division of Women's Health, Brigham and Women's Hospital, Boston, MA, USA; ${ }^{3}$ Division of General Internal Medicine, Massachusetts General Hospital, Boston, MA, USA; ${ }^{4}$ Department of Health Policy and Management, Harvard T.H. Chan School of Public Health, Boston, MA, USA; ${ }^{5}$ Division of General Pediatrics, Department of Medicine, Boston Children's Hospital, Boston, MA, USA; 'tanford University School of Medicine and Graduate School of Business, Stanford, CA, USA.
\end{abstract}

BACKGROUND: Inadequate diagnostic evaluations of breast lumps and rectal bleeding in primary care are an important source of medical errors. Delays appear particularly common in evaluation of rectal bleeding. Comparing pursuit and completion of diagnostic testing for these two conditions within the same practice settings could help highlight barriers and inform interventions.

OBJECTIVES: To examine processes undertaken for diagnostic evaluations of breast lumps and rectal bleeding within the same practices and to compare them with regard to (a) the likelihood that diagnostic tests are ordered according to guidelines and (b) the timeliness of order placement and completion.

DESIGN: A retrospective cohort study using explicit chart abstraction methods.

PARTICIPANTS: Three hundred women aged 30-80 presenting with breast lumps and 300 men and women aged 40-80 years presenting with rectal bleeding to 15 academically affiliated primary care practices, 2012-2016.

MAIN MEASURES: Rates and timing of test ordering and completion and patterns of visits and communications.

KEY RESULTS: At initial presentation, physicians ordered recommended imaging or procedures at higher rates for patients with breast lumps compared to those with rectal bleeding (97\% vs. $86 \%$ of patients recommended to receive imaging or endoscopy; $p<0.01)$. Most (90\%) patients with breast lumps completed recommended diagnostic testing within 1 month, versus $31 \%$ of patients with rectal bleeding $(p<0.01)$. By 1 year, $7 \%$ of patients with breast lumps had not completed indicated imaging, versus $27 \%$ of those with rectal bleeding. Patients with breast lumps had fewer subsequent primary care visits related or unrelated to their symptom and had fewer related communications with specialists.

Prior Presentations None.

Electronic supplementary material The online version of this article (https://doi.org/10.1007/s11606-019-05003-9) contains supplementary material, which is available to authorized users.

Received May 12, 2018

Revised November 6, 2018

Accepted March 19, 2019

Published online April 22, 2019
LIMITATIONS: The study relied on documented care, and findings may be most generalizable to academically affiliated institutions.

CONCLUSIONS: Diagnostic processes for rectal bleeding were less frequently guideline-concordant and timely than those for breast lumps. The largest discrepancies occurred in initial ordering of indicated tests and the timeliness of test completion.

KEY WORDS: breast lumps; rectal bleeding; primary care; care quality; diagnosis.

J Gen Intern Med 34(7):1146-53

DOI: $10.1007 / \mathrm{s} 11606-019-05003-9$

(c) Society of General Internal Medicine 2019

\section{INTRODUCTION}

Breast and colorectal cancers are leading causes of cancerrelated deaths in the USA. ${ }^{1}$ Breast lumps and rectal bleeding are the most common presenting symptoms of breast and colorectal cancers, respectively. ${ }^{2-5}$ Although these symptoms are most often benign, early detection of cancer through evaluation of such "red flag" symptoms may increase the likelihood of patient survival. ${ }^{6-8}$ Thus, most available clinical guidelines and decision support tools recommend early diagnostic evaluation for cancer when a patient presents with either concern. ${ }^{9-11}$

Delayed or inadequate evaluation of cancer symptoms in the ambulatory care setting is a leading cause of medical error and malpractice lawsuits. ${ }^{12-14}$ The quality of diagnostic evaluation is also increasingly a focus of ambulatory quality improvement efforts. $8,15,16$ Variable quality has been noted in primary care evaluations for both breast lumps ${ }^{17}$ and rectal bleeding. ${ }^{15}, 18,19$ However, breast lump and rectal bleeding evaluations have rarely been examined within the same practices, limiting investigation into potential differences and contributing factors. ${ }^{16,20}$ One trial of patient navigation in 11 clinics found that patients with rectal bleeding or positive fecal occult blood tests were less likely to achieve diagnostic resolution than patients with breast concerns. ${ }^{16}$ Another study examining the impact of clinicians' personalities 
found lower likelihood of extended clinical evaluation for rectal bleeding compared with breast lumps. ${ }^{20}$ However, neither study examined the specific points at which differences in diagnostic processes occurred, factors contributing to differences, or the appropriateness of the evaluations in relation to clinical guidelines.

Characterizing where in the diagnostic pathway differences occur, and factors affecting those differences, could illuminate barriers to more timely cancer diagnoses and guide future interventions. We previously found that only half of patients presenting with rectal bleeding within 15 primary care practices received recommended diagnostic evaluation. ${ }^{15}$ The goal of the current study was to compare the quality of diagnostic evaluations performed for patients with rectal bleeding to those performed for patients with breast lumps in those same 15 practices in order to understand how the practices performed on different diagnostic processes and determine the extent to which the evaluation of rectal bleeding may present unique challenges. Specifically, we compare the degree to which patients with a breast lump or with rectal bleeding receive diagnostic evaluations concordant with available decision support tools ${ }^{10,21}$ and the timeliness of completion of these evaluations. We also examine patient factors and process characteristics that may contribute to observed differences.

\section{METHODS}

\section{Study Design}

We conducted a retrospective cohort study of 600 patients presenting to primary care with a chief complaint of either a breast lump $(n=300)$ or rectal bleeding $(n=300)$ between 2012 and 2016. For both conditions, we studied the same 15 primary care practices and used explicit chart abstraction methods to gather information about the diagnostic evaluation process. The institutional review board at the Harvard T.H. Chan School of Public Health approved this study.

\section{Study Setting}

Our study was conducted within 15 primary care practices affiliated with Harvard Medical School. These practices participated in two learning collaboratives between 2012 and 2016: the first aimed to establish or strengthen team-based care (2012-2014), and the second (2014-2016) aimed to improve preventive cancer screening processes for breast and colorectal cancer. ${ }^{15,22,23}$

\section{Data Sources and Study Population}

We combined chart-abstracted data with administrative information available in each practice's electronic health record. We used International Classification of Disease (ICD) Versions 9 and 10, combined with confirmation of presenting symptoms in the medical record, to randomly sample 20 patients per condition from each practice across the four study years for a total of 300 patients presenting with a breast lump and 300 patients presenting with rectal bleeding (Appendix Tables 1 and 2). We oversampled patients with Medicaid insurance, selecting equal numbers of Medicaid-insured and non-Medicaid patients, in order to evaluate differences in care processes between these groups.

For patients presenting with breast lumps, we included women aged 30-80 and excluded patients with a history of breast cancer and patients for whom the primary care physician could not detect an abnormality on clinical breast exam. For patients presenting with rectal bleeding, we included adults aged $40-80$ years and excluded patients with a personal history of colorectal cancer or certain gastrointestinal-related conditions (inflammatory bowel disease, Lynch syndrome) (Appendix Tables 1 and 2). We included patients whose charts confirmed that they presented with a breast lump or rectal bleeding and who remained active patients for the full 365-day observation period.

\section{Chart Abstraction}

Instrument Development. We developed two chart abstraction instruments based on decision support tools from the Controlled Risk Insurance Company (CRICO) (Appendix Figures 1 and 2). ${ }^{10,21}$ For patients with breast lumps, we used the "CRICO Breast Care Management Algorithm" to define recommended diagnostic procedures; this tool recommends diagnostic mammogram and ultrasound for all patients aged 30 or older presenting to primary care with a palpable breast mass. ${ }^{21}$ For patients with rectal bleeding, we used CRICO's "Prevention and Early Detection of Colorectal Cancer" tool. ${ }^{10}$ Details of the rectal bleeding chart abstraction instrument were published previously. ${ }^{15}$

Both instruments captured information from initial primary care presentation until the patient received communication of the results of indicated diagnostic imaging and/or procedures, or 1 year, whichever occurred first. Each instrument gathered general and chief complaint-specific information including (1) patient demographics, (2) screening history, (3) signs, symptoms, and personal characteristics related to the chief complaint (e.g., history of possible non-cancer causes such as breast cysts, hemorrhoids, or diverticulosis, and selected risk factors for/symptoms suggestive of breast cancer (prior breast biopsy, history of BRCA $1 / 2$ mutation, reproductive risk factors) or colorectal cancer (anemia, weight loss, changes in bowel habits, history of colonic adenoma)), (4) family history, (5) other medical conditions, (6) physical exam findings, and (7) care plan (e.g., diagnostic imaging orders, referrals). We then captured follow-up care, including subsequent primary care and specialist visits, and inter-visit communications (e.g., telephone calls and letters). Data were collected independently by four research assistants (CC, KAN, MB, JC). We dually abstracted $10 \%$ of charts $(n=60)$, yielding a Cohen's kappa $=$ 0.95 . Any questions or disagreements were adjudicated by primary care physicians (SP, LP, ATC).

\section{Outcome Variables}

For this study, our main outcomes of interest were (1) frequency and timing of orders for diagnostic tests recommended by 
CRICO guidelines; (2) timeframe within which patients received the ordered tests; (3) frequency of face-to-face visits or inter-visit communications related and unrelated to the patient's symptom after the initial visit; and (4) odds of clinicians ordering recommended tests, and patients receiving ordered tests, adjusted for patient characteristics and patientprovider communication patterns.

\section{Data Analysis}

We used descriptive statistics to compare patient characteristics, testing rates, and visit patterns for patients with breast lumps to patients with rectal bleeding. We used Pearson's chisquare tests when comparing binary or categorical variables, Student's $t$ tests to compare continuous variables, and KaplanMeier curves to illustrate time between initial presentation and initial order placement, and time to order completion. We calculated rates for face-to-face visits bimonthly period and inter-visit communications per bimonthly period. We built two multivariate logistic regression models that adjusted for patient demographic and clinical characteristics. The first model included all patients for whom CRICO guidelines would recommend testing, in order to assess patient and practice factors associated with ordering of recommended tests. The second model included patients who had test orders placed, in order to examine factors associated with receipt of ordered tests. All analyses were conducted with STATA 14 (StataCorp, 2015. Stata Statistical Software: Release 14. College Station, TX: StataCorp LP).

\section{RESULTS}

\section{Patient Characteristics}

Consistent with our eligibility criteria, patients presenting with a chief complaint of a breast lump were significantly younger (breast lump, $47 \pm 11$ years; rectal bleeding, $56 \pm 10$ years, $p<0.01$ ) and had fewer comorbidities (among patients with a breast lump, the proportion with a Charlson Comorbidity Index of $\geq 1$ was $14 \%$ versus $31 \%$ among those with rectal bleeding; $p<0.01$ ) (Table 1). Among patients with a breast lump, $13 \%$ had a documented past non-cancer diagnosis that could explain their symptom versus $50 \%$ of those with rectal bleeding. Thirty-two percent of patients with a breast lump had at least one documented risk factor for breast cancer versus $50 \%$ of those with rectal bleeding. Sixty percent of breast lump patients and $27 \%$ of rectal bleeding patients had none of the identified non-cancer diagnoses and none of the selected cancer risk factors documented in their charts.

\section{Recommended Diagnostic Evaluation}

All patients with a breast lump and $89 \%$ of the patients with rectal bleeding should have been evaluated with diagnostic breast imaging or endoscopy, respectively, based on CRICO guidelines (Table 2). Of those who would have been recommended to have testing, patients presenting with breast lumps had diagnostic tests ordered more frequently than those with rectal bleeding (88\% versus $73 \%, p<0.01)$. More recommended diagnostic mammography orders for patients with breast lumps occurred at initial presentation than diagnostic endoscopy orders for rectal bleeding (Table 2). For patients with breast lumps who had imaging ordered, $97 \%$ had their diagnostic order placed at initial presentation. In contrast, among patients presenting with rectal bleeding who had orders placed, $86 \%$ had their diagnostic order placed at initial presentation while $14 \%$ had orders placed over the subsequent month and year $(p<0.01)$.

Diagnostic test orders for patients presenting with rectal bleeding took longer to be completed than for patients with breast lumps (Table 2, Fig. 1). While 90\% of patients with breast lumps who had diagnostic imaging ordered had imaging completed within 90 days, only $57 \%$ of those with rectal bleeding had their ordered endoscopy completed within 90 days. By 12 months, $73 \%$ of rectal bleeding patients for whom endoscopy was ordered had the study performed, versus $93 \%$ of patients with breast lumps for whom diagnostic mammograms were ordered. Overall, among patients for whom diagnostic testing was recommended by CRICO, 246 $(82 \%)$ of patients with breast lumps and $144(55 \%)$ with rectal bleeding had the test completed within 1 year. By the end of our abstraction period, 17 patients with breast lumps and 1 patient with rectal bleeding had been diagnosed with cancer.

\section{Visit and Communication Patterns}

Table 3 describes patients' visits and communication patterns following initial presentation to primary care with a breast lump or rectal bleeding, among those who would have been recommended for further evaluation by CRICO guidelines. On average, patients with breast lumps had fewer related follow-up primary care visits during the study period compared with patients with rectal bleeding (breast lumps, $0.5 \pm$ 0.8 visits; rectal bleeding, $0.9 \pm 1.2$ visits, $p<0.01$ ). The number of related follow-up visits to specialists was similar in both groups $(0.6 \pm 0.9$ versus $0.5 \pm 0.9, p=0.20)$. Among those who had intra-visit communications, patients with breast lumps had non-significantly fewer non-face-to-face communications with both primary care $(0.6 \pm 1.1$ versus $0.8 \pm 1.2$, $p=0.07)$ and significantly fewer communications with specialists compared with patients with rectal bleeding $(0.1 \pm 0.5$ versus $0.5 \pm 0.8, p<0.01$ ). Patients with breast lumps also had fewer visits and non-face-to-face communications with both primary care and specialists that were unrelated to their chief complaint.

\section{Factors Associated with Diagnostic Evaluation Orders and Completion}

In the multivariate logistic regression models examining the likelihood of order placement among patients who should have had diagnostic mammography or endoscopy according 
Table 1 Study Population by Chief Complaint

\begin{tabular}{|c|c|c|c|}
\hline & $\begin{array}{l}\text { Breast lump } \\
N=\mathbf{3 0 0}\end{array}$ & $\begin{array}{l}\text { Rectal bleeding } \\
N=\mathbf{3 0 0}\end{array}$ & $p$ value* \\
\hline \multicolumn{4}{|l|}{ General patient characteristics } \\
\hline Age in years, mean \pm standard deviation $(\mathrm{SD})$ & $47 \pm 11$ & $56 \pm 10$ & $<0.01$ \\
\hline Age $30-39$ years, $\%(n)$ & $25 \%(76)$ & - & NA \\
\hline $40-49$ & $42 \%(127)$ & $33 \%(98)$ & \\
\hline $50-59$ & $18 \%(53)$ & $35 \%(106)$ & \\
\hline $60-69$ & $9 \%(27)$ & $22 \%(65)$ & \\
\hline$\geq 70$ & $6 \%(17)$ & $10 \%(31)$ & \\
\hline Female $(\%)$ & $100 \%(300)$ & $45 \%$ & $<0.01$ \\
\hline White race/ethnicity, \% (n) & $36 \%(108)$ & $37 \%(111)$ & \\
\hline Hispanic/Latino & $29 \%(88)$ & $27 \%(80)$ & \\
\hline African American & $21 \%(63)$ & $21 \%(63)$ & 0.19 \\
\hline Asian/Pacific Islander & $3 \%(10)$ & $8 \%(23)$ & \\
\hline Other/unknown & $10 \%(24)$ & $7 \%(23)$ & \\
\hline English as primary language, $\%(n)$ & $64 \%(191)$ & $63 \%(188)$ & 0.80 \\
\hline Commercial insurance $(\%)$ & $43 \%(127)$ & $38 \%(114)$ & \\
\hline Medicare & $7 \%(21)$ & $12 \%(36)$ & 0.99 \\
\hline Medicaid & $50 \%(150)$ & $50 \%(150)$ & \\
\hline Charlson Comorbidity Index $\geq 1, \%(n)$ & $14 \%(43)$ & $31 \%(94)$ & $<0.01$ \\
\hline \multicolumn{4}{|l|}{$\begin{array}{l}\text { Documented prior screening, benign disease, } \\
\text { and cancer risk factors }\end{array}$} \\
\hline $\begin{array}{l}\text { Prior corresponding }{ }^{\dagger} \text { screening mammogram } \\
\text { or colonoscopy documented, \% }(n)\end{array}$ & $58 \%(175)$ & $48 \%(144)$ & 0.01 \\
\hline $\begin{array}{l}\text { Among those with prior corresponding }{ }^{\dagger} \text { screening } \\
\text { test, time in years since prior test, mean } \pm \text { SD }\end{array}$ & $1.7 \pm 2.7$ & $4.2 \pm 2.8$ & NA \\
\hline $\begin{array}{l}\text { No documented cancer risk factors or characteristics } \\
\text { suggestive of non-cancer diagnosis, \% }(n)\end{array}$ & $60 \%(181)$ & $27 \%(80)$ & $<0.01$ \\
\hline$\geq 1$ characteristic suggestive of non-cancer diagnosis ${ }^{\star}, \%(n)$ & $13 \%(38)$ & $50 \%(149)$ & NA \\
\hline$\geq 1$ suggestive of cancer, $\%(n)$ & $32 \%(97)$ & $50 \%(150)$ & \\
\hline \multicolumn{4}{|l|}{ For breast lump presentation } \\
\hline Family history of breast cancer & $20 \%(66)$ & _- & \\
\hline Personal history of breast biopsy & $9 \%(25)$ & _ & \\
\hline Known carrier of BRCA mutation & $0.3 \%(1)$ & _- & \\
\hline Reproductive risk ${ }^{\S}$ & $8 \%(26)$ & _- & \\
\hline \multicolumn{4}{|l|}{ For rectal bleeding presentation } \\
\hline Family history of colorectal cancer & - & $3 \%(10)$ & \\
\hline Personal history of adenoma & _ & $10 \%(27)$ & \\
\hline Anemia & - & $7 \%(21)$ & \\
\hline Unintentional weight loss & - & $2 \%(6)$ & \\
\hline Change in bowel habits & & $57 \%(112)$ & \\
\hline
\end{tabular}

*According to Student's $t$ tests for continuous variables and chi-squared tests for categorical ones

+Corresponding cancer indicates breast cancer for patients presenting with breast lump and colorectal cancer for ones presenting with rectal bleeding $\mp$ Characteristic that is suggestive of potential non-breast cancer etiology of a lump would be personal history of breast cyst(s). Characteristic suggestive of potential non-colorectal cancer diagnosis would be history or physical exam finding of either hemorrhoids or diverticulosis

${ }^{\xi}$ Nulliparity, menarche $\leq 12$ years of age, first birth $\geq 30$ years of age; no patients had more than 5 years of combined hormone replacement therapy. NA not applicable

Table 2 Guideline Concordance and Timing of Diagnostic Mammogram/Colonoscopy Orders

\begin{tabular}{|c|c|c|c|}
\hline & Breast lump & Rectal bleeding & $p$ value \\
\hline $\begin{array}{l}\text { Of total presenting, } \%(N) \text { for whom clinical guidelines recommend } \\
\text { mammogram for breast lump or colonoscopy for rectal bleeding }\end{array}$ & $100 \%(300)$ & $89 \%(268)$ & $<0.01$ \\
\hline $\begin{array}{l}\text { Among patients for whom order is placed, timing of order placement } \\
\text { relative to presentation* }\end{array}$ & $N=264$ & $N=196$ & \\
\hline At initial presentation & $97 \%(257)$ & $86 \%(169)$ & $<0.01$ \\
\hline Between 61 and 365 days after presentation & $1 \%(2)$ & $8 \%(15)$ & \\
\hline Among patients for whom order is placed, timing of order completion* & $N=264$ & $N=196$ & \\
\hline Within 30 days of order placement & $84 \%(221)$ & $31 \%(61)$ & \\
\hline Between 31 and 90 days after order placement & $6 \%(17)$ & $26 \%(51)$ & $<0.01$ \\
\hline Between 91 and 365 days after order placement & $3 \%(8)$ & $16 \%(32)$ & \\
\hline Not completed within 365 days & $7 \%(18)$ & $27 \%(52)$ & \\
\hline
\end{tabular}

*Orders correspond to mammograms for chief complaints of breast lump and to colonoscopies for chief complaints of rectal bleeding †Using chi-squared test

${ }^{a}$ Controlled Risk Insurance Company. CRICO Breast Care Management Algorithm: A Decision Support Tool.; 2014.

${ }^{b}$ Controlled Risk Insurance Company. Prevention \& Early Detection of Colorectal Cancer: A CRICO Decision Support Tool.; 2014. 


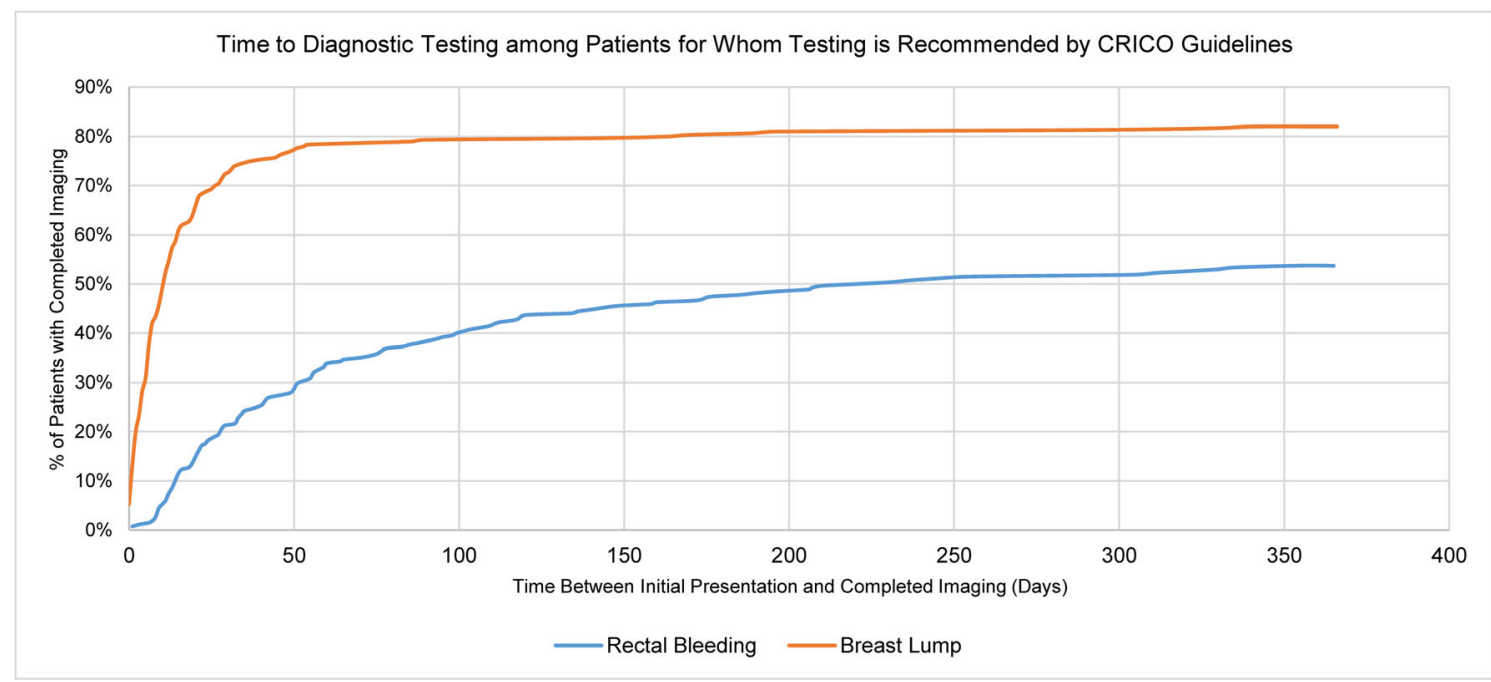

Figure 1. Time between initial presentation and completion of diagnostic testing, among patients for whom diagnostic testing is recommended by CRICO guidelines.

to CRICO, patients with breast lumps were significantly more likely to have had orders placed for recommended tests, compared with patients with rectal bleeding (OR 3.76, 95\% CI $[1.77,8.03])$ when adjusting for patient characteristics, prior screening, and visit patterns. In the model examining the likelihood of order completion, patients with breast lumps were more likely to have ordered tests completed (OR 7.06, $95 \%$ CI $[2.89,17.28]$ ) (Table 4). Patients with no prior documented screening or whose last relevant screening test was over 5 years ago were more likely to have a recommended diagnostic test ordered (OR 2.34, 95\% CI [1.15, 4.79] and OR $3.28,95 \%$ CI [1.21, 8.87], respectively). Documented risk factors or symptoms concerning for cancer were not associated with ordering or receipt of recommended testing. However, a history suggestive of a non-cancer diagnosis significantly decreased the likelihood that an indicated test would be

Table 3 Visits and Communications Within 1 Year Among Those for Whom Diagnostic Imaging/Procedures Were Recommended

\begin{tabular}{|c|c|c|c|}
\hline $\begin{array}{l}\text { Number of visits and } \\
\text { communications, mean } \pm \text { SD }\end{array}$ & $\begin{array}{l}\text { Breast } \\
\text { lump } \\
N=\mathbf{3 0 0}\end{array}$ & $\begin{array}{l}\text { Rectal } \\
\text { bleeding } \\
N=\mathbf{2 6 8}\end{array}$ & $p$ \\
\hline \multicolumn{4}{|l|}{ Related to chief complaint } \\
\hline \multicolumn{4}{|l|}{ Primary Care } \\
\hline Face-to-face visits & $0.5 \pm 0.8$ & $0.9 \pm 1.2$ & $<0.01$ \\
\hline Intra-visit communications & $0.6 \pm$ & 08 & 0.07 \\
\hline \multicolumn{4}{|c|}{ Corresponding* specialist visits } \\
\hline Face-to-face visits & $0.6 \pm 0.9$ & 0.9 & 0.20 \\
\hline Intra-visit communications & $0.1 \pm 0.5$ & $0.5 \pm 0.8$ & $<0.0$ \\
\hline \multicolumn{4}{|c|}{ Unrelated to corresponding* chief complaint } \\
\hline \multicolumn{4}{|l|}{ Primary care } \\
\hline Face-to-face visits & $1.1 \pm 1.8$ & & $<0.01$ \\
\hline Intra-visit communication & $1.8 \pm 3.5$ & 3.2 & $<0.0$ \\
\hline \multicolumn{4}{|c|}{ Corresponding* specialist visits } \\
\hline Face-to-face visits & $1.8 \pm 4.1$ & 3.3 & $<0.01$ \\
\hline Intra-visit communications & $0.5 \pm 1.5$ & $4.8 \pm 6.7$ & $<0.01$ \\
\hline
\end{tabular}

*Specialist visits related to corresponding chief complaint for patients presenting with breast lump include those to the Emergency Department, surgery, oncology, and breast center. Specialist visits related to corresponding chief complaint of rectal bleeding include Emergency

Department, gastroenterology, surgery, oncology, and hematology ordered (OR 0.60, 95\% CI [0.37, 0.98]). Age, gender, race/ethnicity, Charlson Comorbidity Index, number of unrelated visits, and insurance were not associated with order or receipt of testing.

\section{DISCUSSION}

In this study of 15 primary care practices, we found that rates of both ordering and receipt of recommended diagnostic tests were lower among patients with rectal bleeding compared with patients with breast lumps. In addition, among those for whom diagnostic tests were ordered, there were longer delays in receipt of testing among patients with rectal bleeding compared with those with breast lumps. We have previously described relatively high rates of guideline non-concordance in diagnostic evaluation of rectal bleeding in these practices ${ }^{15}$ consistent with findings from other settings. ${ }^{16,18,20,26,28}$ This analysis adds to the literature by demonstrating striking differences in both guideline concordance and timeliness of processes for rectal bleeding and breast lumps within the same practices.

Our findings highlight differences in evaluation pathways for rectal bleeding and breast lumps and suggest barriers unique to rectal bleeding. First, providers tended to delay more in initially ordering indicated diagnostic endoscopies for patients with rectal bleeding. There are several potential explanations for this. Providers may have less familiarity with guidelines for rectal bleeding. Although decision tools such as CRICO are recommended as a strategy to mitigate diagnostic errors, making them available and referenced at the point of care is challenging. ${ }^{29}$ In addition, clinicians may have a lower degree of concern about missing colorectal cancer presenting as rectal bleeding compared with missing a breast cancer. ${ }^{20}$ This may reflect more experience diagnosing breast cancer following a breast lump, as suggested in the higher number of breast cancers diagnosed. It was notable that patients with rectal bleeding had more symptom-related face-to-face visits 
Table 4 Adjusted Odds of Recommended Diagnostic Image/Procedure Order Placement and Completion

\begin{tabular}{|c|c|c|c|c|c|c|}
\hline & \multicolumn{6}{|c|}{ Recommended diagnostic imaging/procedure } \\
\hline & Odds ratio & $95 \% \mathrm{CI}$ & $p$ value & Odds ratio & $95 \% \mathrm{CI}$ & $p$ value \\
\hline $\begin{array}{l}\text { Chief complaint, rectal bleeding as referent } \\
\text { Breast Lump }\end{array}$ & 3.37 & $1.58,7.19$ & $<0.01$ & 7.06 & $2.89,17.28$ & $<0.01$ \\
\hline Sex, male as referent & & & & & & \\
\hline $\begin{array}{l}\text { Female } \\
\text { Race/ethnicity, White as referent }\end{array}$ & 1.12 & $0.62,2.02$ & 0.70 & 0.81 & $0.40,1.61$ & 0.54 \\
\hline $\begin{array}{l}\text { Non-White } \\
\text { Primary }\end{array}$ & 1.59 & $0.94,2.70$ & 0.08 & 1.48 & $0.79,2.77$ & 0.22 \\
\hline $\begin{array}{l}\text { Non-English } \\
\text { Insurance, commercial or Medicare as referent }\end{array}$ & 0.86 & $0.49,1.48$ & 0.58 & 1.62 & $0.83,3.13$ & 0.16 \\
\hline $2-5$ years & 1.56 & $0.68,3.57$ & 0.30 & 0.70 & $0.22,2.21$ & 0.55 \\
\hline$>5$ years & 3.50 & $1.29,9.51$ & 0.01 & 1.37 & $0.38,4.95$ & 0.63 \\
\hline Not documented or no prior screening & 2.49 & $1.21,5.10$ & 0.01 & 0.88 & $0.32,2.45$ & 0.81 \\
\hline $\begin{array}{l}\text { Risk factor for corresponding* cancer, zero as } \\
\geq 1\end{array}$ & $\begin{array}{l}\text { referent } \\
0.93\end{array}$ & $0.59,1.47$ & 0.76 & 1.23 & $0.69,2.18$ & 0.49 \\
\hline $\begin{array}{l}\text { Risk factor suggestive of non-cancer diagnosis, } \\
\geq 1\end{array}$ & $\begin{array}{l}\text { zero as referent } \\
0.58\end{array}$ & $0.35,0.95$ & 0.03 & 1.38 & $0.73,2.61$ & 0.32 \\
\hline \multicolumn{7}{|c|}{ Number of face-to-face visits unrelated to corresponding chief complaint, zero as referent } \\
\hline$\geq 1$ & 0.42 & $0.25,0.72$ & $<0.01$ & 0.68 & $0.37,1.26$ & 0.22 \\
\hline
\end{tabular}

following initial presentation. Although this could reflect these patients' higher comorbidity burden, or more adoption of surveillance prior to testing, it also may reflect enhanced complexity of decision-making about and planning for endoscopy. Patients with rectal bleeding also had more unrelated primary care visits, which could reflect competing priorities for providers and patients - though in multivariate analyses, higher numbers of visits did not significantly decrease the likelihood of test order placement.

Our multivariate models suggest that providers considered patients' risk for cancer in ordering tests. For example, patients with more recent prior screening and patients with histories of benign conditions that can cause breast lumps or rectal bleeding were less likely to have diagnostic tests ordered. However, previous benign disease is not considered to modify the need for diagnostic testing in the CRICO rules, and having a colonoscopy in the past 2 years only modestly alters CRICO's recommendations for endoscopy in cases of rectal bleeding. Further, we are not aware of other guidelines that provide detailed risk stratification among patients with rectal bleeding. Limited evidence suggests that, particularly in patients over 50 , rectal bleeding alone has a sufficient positive predictive value to merit further evaluation for cancer. ${ }^{3}$ Further research into modifying factors is needed to guide a more individualized approach.

Once colonoscopies were ordered, more patients with rectal bleeding experienced clinically significant delays in receipt of testing compared with patients with breast lumps for whom breast imaging was ordered. This could reflect logistical barriers to test completion for patients with rectal bleeding. Endoscopy is more invasive and time-intensive than a mammogram, and typically takes longer to schedule, leaving more opportunity for attrition and gaps in care. ${ }^{25,} 28,{ }^{30}$ Patient factors also may contribute. Although many patients find mammograms uncomfortable, colonoscopy preparation and procedures are typically considered more unpleasant and require patients to be escorted home, which could limit test adherence. Nationwide, rates of adherence to colonoscopy screening are lower than rates of mammography. ${ }^{24}$ Patients may also be more aware of and concerned about breast cancer than colorectal cancer.

There is robust literature examining the factors involved in follow-up of abnormal screening tests. ${ }^{26}$ Our study suggests the need for closer examination of factors involved in evaluation of cancer "red flag" symptoms, especially rectal bleeding. Primary care providers evaluating patients with rectal bleeding appear to engage in risk stratification that is not well-supported by existing research or guidelines. However, given the logistical and resource barriers to endoscopic evaluation, and risks associated with endoscopy, research to inform more tailored guidelines may be warranted. Our findings also suggest the need for attention to barriers to completion of diagnostic endoscopy. Streamlined referral pathways for symptomatic patients, facilitation of multidisciplinary 
communication, and patient navigation could be important tools to expedite endoscopy. Of note, the few studies of patient navigators for expediting diagnostic evaluations of "red flag" symptoms have had mixed results. ${ }^{16}$

Our study has several limitations. First, it focused on ordering and receipt of diagnostic tests recommended by the CRICO decision support tools. In reality, receipt of a mammogram or a colonoscopy could be the first step in a potentially complex process that would include results communication and potentially more testing. ${ }^{26}$ This could be particularly true for mammograms since a separate appointment is typically required for biopsies. Follow-up of abnormal mammograms is a known quality challenge ${ }^{27}$ that we did not assess, and we did not focus on results communication, since documentation of this was unreliable. Second, our ability to examine the factors contributing to each step of the diagnostic process was limited by the information available in the clinical record. Information on clinical history was limited, particularly the presence of non-cancer diagnoses that could explain the presenting symptom and cancer risk factors. We did not examine the role of patient preferences and nonadherence, which likely disproportionately impact uptake of endoscopy. We also could not usually identify specific system- and provider-related challenges to scheduling tests. Lastly, our study describes diagnostic processes at 15 Harvard-affiliated practices in the Boston area. Although the practices were relatively diverse in their patient populations and located both in hospitals and communities, they are not generalizable to other geographic and nonacademic settings.

In summary, even in practices that perform relatively well in prompt evaluation of breast lumps, there is a need for improved adherence to recommended diagnostic evaluation for rectal bleeding. While differences in clinical decision-making appeared to contribute to lower rates of test ordering for rectal bleeding, and limited guideline awareness could be a factor, logistical and health system-related barriers probably play a significant role in test completion. These issues require further evaluation to inform quality improvement.

Contributors: Marnie Blalock, BS; Jenny Chen, BA; Sahitya Raja, MMSc.

Corresponding Author: Lydia E. Pace, MD, MPH; Division of Women's Health Brigham and Women's Hospital, OBC 3-34, 75 Francis Street, Boston, MA 02115-9950, USA (e-mail: lpace@bwh. harvard.edu).

Funders This study was funded by the CRICO Risk Management Foundation. LEP was supported by an American Cancer Society Career Development Award for Primary Care Physicians (Grant 130741-CCCDA-17-072-01-CCCDA) and a National Cancer Institute K07 Career Development Award (Grant 1K07CA215819-01A1).

\section{Compliance with Ethical Standards:}

The institutional review board at the Harvard T.H. Chan School of Public Health approved this study.
Conflict of Interest: The authors declare that they do not have a conflict of interest.

\section{REFERENCES}

1. Howlader, N. et al. SEER Cancer Statistics Review, 1975-2014. (2017).

2. Jemal, A. et al. Cancer; Statistics , 2006. (2006). doi:https://doi.org/10. 3322/canjclin.54.1.8

3. Astin, M., Griffin, T., Neal, R. D., Rose, P. \& Hamilton, W. The diagnostic value of symptoms for colorectal cancer in primary care: a systematic review. Br J Gen Pract 61, 231-243 (2011).

4. Talley, N. J. \& Jones, M. Self-reported rectal bleeding in a United States community: prevalence, risk factors, and health care seeking. Am J Gastroenterol 93, 2179-2183 (1998).

5. Barlow, w. E. et al. Performance of diagnostic mammography for women with signs or symptoms of breast cancer. J Natl Cancer Inst 94, 1151-9 (2002).

6. Thompson, M. R., Asiimwe, A., Flashman, K. \& Tsavellas, G. Is earlier referral and investigation of bowel cancer patients presenting with rectal bleeding associated with better survival? Color Dis 13, 1242-1248 (2011).

7. Tørring, M. L. et al. Evidence of advanced stage colorectal cancer with longer diagnostic intervals: a pooled analysis of seven primary care cohorts comprising 11720 patients in five countries. Br $J$ Cancer 117, 888-897 (2017).

8. Neal, R. D. et al. Is increased time to diagnosis and treatment in symptomatic cancer associated with poorer outcomes? Systematic review. Br J Cancer 112, S92-S107 (2015)

9. American Cancer Society. Early Detection, Diagnosis, and Staging. (2016). Available at: https://www.cancer.org/content/dam/CRC/PDF/ Public/8606.00.pdf. (Accessed: 28th December 2018)

10. Controlled Risk Insurance Company. Prevention \& Early Detection of Colorectal Cancer: A CRICO Decision Support Tool. (2014). Available at: https://www.rmf.harvard.edu/guidescolorectal. (Accessed: 28th December 2018)

11. Controlled Risk Insurance Company. CRICO Breast Care Management Algorithm: A Decision Support Tool. (2014). Available at: https://www.rmf. harvard.edu/Clinician-Resources/Guidelines-Algorithms/2014/BreastCare-Management-Algorithm. (Accessed: $28^{\text {th }}$ December 2018)

12. Gandhi, T. K. et al. Missed and delayed diagnoses in the ambulatory setting: a study of closed malpractice claims. Ann Intern Med 145, 488496 (2006).

13. Schiff, G. D. et al. Primary Care Closed Claims Experience of Massachusetts Malpractice Insurers. JAMA Intern Med 173, 2063 (2013).

14. Singh, H., Sethi, S., Raber, M. \& Petersen, L. A. Errors in Cancer Diagnosis: Current Understanding and Future Directions. J Clin Oncol 25, 5009-5018 (2007).

15. Percac-Lima, S. et al. Diagnostic Evaluation of Patients Presenting to Primary Care with Rectal Bleeding. J Gen Intern Med (2018). doi:https:// doi.org/10.1007/s11606-017-4273-x

16. Wells, K. J. et al. A cluster randomized trial evaluating the efficacy of patient navigation in improving quality of diagnostic care for patients with breast or colorectal cancer abnormalities. Cancer Epidemiol Biomark Prev 21, 1664-72 (2012).

17. Haas, J. S., Cook, E. F., Puopolo, A. L., Burstin, H. R. \& Brennan, T. A. Differences in the quality of care for women with an abnormal mammogram or breast complaint. J Gen Intern Med 15, 321-8 (2000).

18. Weingart, $\mathbf{S}$. N. et al. Delayed Workup of Rectal Bleeding in Adult Primary Care: Examining Process-of-Care Failures. Jt Comm $J$ Qual Patient Saf 43, 32-40 (2017).

19. Weingart, $\mathbf{S}$. $\mathbf{N}$. et al. Working up rectal bleeding in adult primary care practices. J Eval Clin Pract (2016). doi:https://doi.org/10.1111/jep. 12596

20. Allen, A. S., Orav, E. J., Lee, T. H. \& Sequist, T. D. Clinician personality and the evaluation of higher-risk patient symptoms. J Patient Saf 7, 1226 (2011).

21. Controlled Risk Insurance Company. CRICO Breast Care Management Algorithm: A Decision Support Tool. (2014).

22. Wu, C. A. et al. A population management system for improving colorectal cancer screening in a primary care setting. $J$ Eval Clin Pract 22, 319-328 (2016). 
23. Bitton, A. et al. The Harvard Medical School Academic Innovations Collaborative: transforming primary care practice and education. Acad Med 89, 1239-44 (2014).

24. Smith, R. et al. Cancer Screening in the United States, 2017: A Review of Current American Cancer Society Guidelines and Current Issues in Cancer Screening. CA Cancer J Clin 67, 100-121 (2017).

25. Green, A. R. et al. Barriers to Screening Colonoscopy for Low-income Latino and White Patients in an Urban Community Health Center. $J$ Gen Intern Med 23, 834-840 (2008).

26. Zapka, J. et al. Factors in Quality Care-The Case of Follow-Up to Abnormal Cancer Screening Tests-Problems in the Steps and Interfaces of Care. JNCI Monogr 2010, 58-71 (2010).

27. Taplin, S. H., Yabroff, K. R. \& Zapka, J. A multilevel research perspective on cancer care delivery: the example of follow-up to an abnormal mammogram. Cancer Epidemiol Biomark Prev 21, 1709-15 (2012).
28. Wahls, T. L. \& Peleg, I. Patient- and system-related barriers for the earlier diagnosis of colorectal cancer. BMC Fam Pract 10, 65 (2009).

29. Poon, E. G., Kachalia, A., Puopolo, A. L., Gandhi, T. K. \& Studdert, D. M. Cognitive Errors and Logistical Breakdowns Contributing to Missed and Delayed Diagnoses of Breast and Colorectal Cancers: A Process Analysis of Closed Malpractice Claims. J Gen Intern Med 27, 1416-1423 (2012).

30. Bhise, v. et al. Patient-Reported Attributions for Missed Colonoscopy Appointments in Two Large Healthcare Systems. Dig Dis Sci 61, 18531861 (2016)

Publisher's Note Springer Nature remains neutral with regard to jurisdictional claims in published maps and institutional affiliations. 